\title{
Microstructural Correlates of Infant Functional Development: Example of the Visual Pathways
}

\author{
Jessica Dubois, ${ }^{1,9}$ Ghislaine Dehaene-Lambertz, ${ }^{2,6,9}$ Catherine Soarès, ${ }^{2,6,9}$ Yann Cointepas, ${ }^{3,9}$ Denis Le Bihan, ${ }^{4,9}$ and \\ Lucie Hertz-Pannier ${ }^{5,7,8,9}$ \\ ${ }^{1}$ Unité de Neuroimagerie Anatomique et Fonctionnelle, Commissariat à l'Energie Atomique (CEA)/Saclay (SAC)/Division des Sciences du Vivant (DSV)/ \\ Institut d'imagerie Biomédicale (I2BM)/Service Hospitalier Frédéric Joliot, 91401 Orsay, France, ${ }^{2}$ Institut National de la Santé et de la Recherche Médicale, \\ U562, ${ }^{3}$ Laboratoire de Neuroimagerie Assistée par Ordinateur, CEA, ${ }^{4}$ NeuroSpin, CEA, and ${ }^{5}$ Laboratoire de Recherche Biomédicale, CEA/SAC/DSV/I2BM/ \\ NeuroSpin, 91191 Gif-sur-Yvette, France, ${ }^{6}$ Assistance Publique/Hôpitaux de Paris (AP-HP), Neuropédiatrie, Centre Hospitalier Universitaire, 94275 Bicêtre, \\ France, ${ }^{7}$ AP-HP, Radiologie Pédiatrique, Hôpital Necker-Enfants Malades, 75015 Paris, France, ${ }^{8}$ Institut National de la Santé et de la Recherche Médicale, \\ U663, Université Paris 5, 75015 Paris, France, and ${ }^{9}$ Institut Fédératif de Recherche, 75 Paris, France
}

The development of cognitive functions during childhood relies on several neuroanatomical maturation processes. Among these processes is myelination of the white matter pathways, which speeds up electrical conduction. Quantitative indices of such structural processes can be obtained in vivo with diffusion tensor imaging (DTI), but their physiological significance remains uncertain. Here, we investigated the microstructural correlates of early functional development by combining DTI and visual event-related potentials (VEPs) in 15 one- to 4-month-old healthy infants. Interindividual variations of the apparent conduction speed, computed from the latency of the first positive VEP wave (P1), were significantly correlated with the infants' age and DTI indices measured in the optic radiations. This demonstrates that fractional anisotropy and transverse diffusivity are structural markers of functionally efficient myelination. Moreover, these indices computed along the optic radiations showed an early wave of maturation in the anterior region, with the posterior region catching up later in development, which suggests two asynchronous fronts of myelination in both the geniculocortical and corticogeniculate fibers. Thus, in addition to microstructural information, DTI provides noninvasive exquisite information on the functional development of the brain in human infants.

Key words: myelination; DTI; tractography; ERP; VEP; P1 latency; conduction speed

\section{Introduction}

Because of development of neuroimaging, it has become possible to study the structural changes underlying the rapid development of the human brain in vivo. The first studies using positron emission tomographic scanning (Chugani and Phelps, 1986), single photon emission computed tomography (Chiron et al., 1992), and conventional magnetic resonance imaging (MRI) (Paus et al., 2001) have confirmed postmortem observations (Yakovlev and Lecours, 1967; Huttenlocher and Dabholkar, 1997) of substantial differences in maturation time courses across the different brain regions. Recent progress in diffusion tensor imaging (DTI), which provides quantitative markers of tissue microstructure (for a review, see Le Bihan et al., 2001), now makes it possible to go from the assessment of regional variations to precise mea-

\footnotetext{
Received Aug. 6, 2007; revised Dec. 20, 2007; accepted Dec. 20, 2007

This work was supported by the McDonnell Foundation. We thank J.-F. Mangin, M. Perrin, C. Poupon, F. Lethimonnier, D. Rivière, E. Duchesnay, and A. Cachia for support on the DTI acquisition and the postprocessing; T. Gliga for help on the VEP experiment; E. Hubbard for his careful reading of this manuscript; T. Brunet (Ravier-Touzard Company) for designing a baby bouncer chair specifically adapted to the head coil; P. Landrieu and F. Brunelle for their support; and ANR for financial support to G.D.-L.

Correspondence should be addressed to Dr. Jessica Dubois, U663, Hôpital Necker-Enfants maladies, 149 rue de Sèvres, 75015 Paris, France. E-mail: jessica.dubois@centraliens.net.

DOI:10.1523/JNEUROSCI.5145-07.2008

Copyright $\odot 2008$ Society for Neuroscience $\quad$ 0270-6474/08/281943-06\$15.00/0
}

sures in individual white matter fascicles isolated by tractography (for a review, see Mori and van Zijl, 2002). Previous studies have demonstrated correlations between age and increases in fractional anisotropy $(\mathrm{FA})$ and decreases in mean $(<D>)$, transverse $\left(\lambda_{\perp}\right)$, and longitudinal $\left(\lambda_{/ /}\right)$diffusivities (for a review, see Neil et al., 2002; Partridge et al., 2004). These indices reflect brain water content, cell proliferation, tract organization, compactness, and myelination (for review, see Beaulieu, 2002), but their precise functional significance remains uncertain. Whereas $\lambda_{/ /}$may reflect axonal organization within a tract, changes in FA and $\lambda_{\perp}$ may predominantly depend on myelination in the first postnatal months (Song et al., 2003; Dubois et al., 2008). Given that myelination increases the speed of electrical conduction along fibers, these latter structural changes should be correlated with improved electrical transmission and a correspondingly decreased latency for event-related potentials (ERPs).

To test this assumption, we examined both the structural and functional development of the visual system between 1 and 4 months of age by recording, in the same healthy infants, whole brain DTI images and electrophysiological responses to visual stimuli. This developmental period is characterized by a fast increase in synaptic density in the visual cortex (Huttenlocher et al., 1982 ) and an intense myelination of visual pathways (Yakovlev and Lecours, 1967). The latency of the first positive wave (P1), the 
a
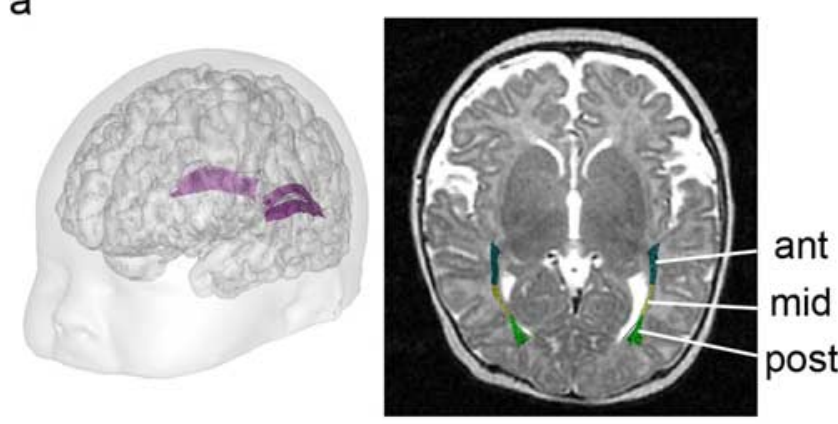

b

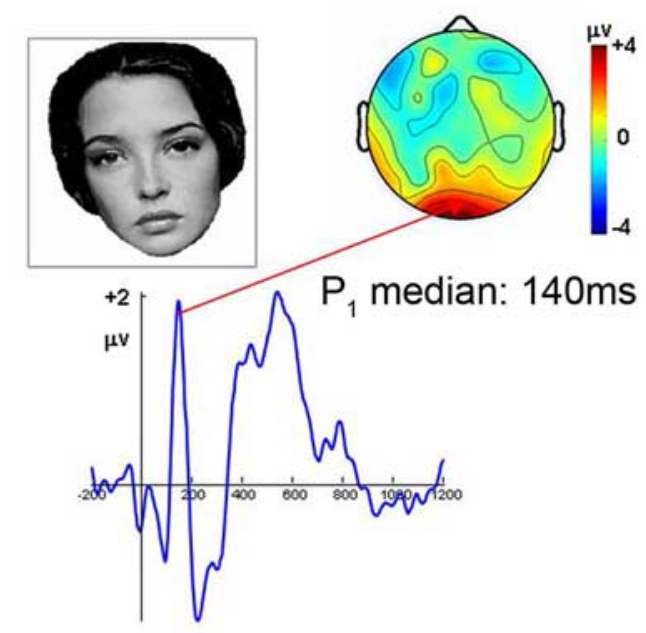

Figure 1. $\boldsymbol{a}$, Reconstruction by tractography of the optic radiations, with three segments. $\boldsymbol{b}$, Visual evoked response to faces: grand-average across all infants measured across six electrodes around $0 z$, and $\mathrm{P} 1$ topographic map at $140 \mathrm{~ms}$. ant, Anterior; mid, middle; post, posterior.

most consistent feature of infants' visual evoked potentials (VEPs), dramatically decreases from $260 \mathrm{~ms}$ in neonates to 110 ms by the age of 4 months (Crognale et al., 1997; McCulloch et al., 1999). Although other developmental processes such as maturation of the retina, lateral geniculate nucleus (LGN), and occipital cortex contribute to this gain in speed, it primarily results from myelination of the visual pathways (Kos-Pietro et al., 1997; Tsuneishi and Casaer, 1997). Thus, we hypothesized that if DTI changes in FA and $\lambda_{\perp}$ were actually structural markers of fibers' myelination in this age range, their values in the optic radiations should be strongly correlated with P1 latency, regardless of the effect of age and with no such correlation in the other white matter pathways. In addition, it should become possible to assess the spatial progression of myelination by studying these indices along the optic radiations across different ages. For the first time, this approach would provide quantitative microstructural correlates of functional development in human infants.

\section{Materials and Methods}

Subjects. Fifteen healthy full-term infants were tested between 5.6 and 17.1 weeks (mean age, $11.2 \pm 3.3$ weeks) after their parents gave written informed consent. Two additional infants were excluded because of insufficient quality of the VEP recordings for one and drowsiness, a factor known to affect P1 latency (Benavente et al., 2005), for the other. This study was approved by the regional ethical committee for biomedical research.

DTI imaging. DTI data have been reported, in part, in a previous study (Dubois et al., 2008).

Data acquisition. The acquisition was performed on a $1.5 \mathrm{~T}$ MRI system (Signa LX; GEMS, Milwaukee, WI). No sedation was used, and particular precautions were taken to reduce exposure to the MRI scanner noise. A diffusion-weighted, spin echo, echo-planar imaging technique was used, with a $700 \mathrm{~s} . \mathrm{mm}^{-2} \mathrm{~b}$ factor and $14-30$ diffusion gradient encoding orientations (echo time/repetition time, $89.6 \mathrm{~ms} / 13.8 \mathrm{~s}$; spatial resolution, $\left.0.94 \times 0.94 \times 2.5 \mathrm{~mm}^{3}\right)$. T2-weighted images were acquired using a fast spin echo sequence to provide anatomical landmarks.

Data postprocessing and analysis. After estimation of the diffusion tensor, maps of FA, $\left\langle D>, \lambda_{\perp}\right.$, and $\lambda_{/ /}$were calculated with BrainVISA software (Cointepas et al., 2003) (http://brainvisa.info). Individual optic radiations were reconstructed by three-dimensional (3D) tractography (Dubois et al., 2008) between two regions positioned at the level of the LGN and the occipital pole (Catani et al., 2002) using a nonlinear algorithm (Perrin et al., 2005), excluding voxels with low FA $(<0.15)$ or high $<D>\left(>2.10^{-3} \mathrm{~mm}^{2} . \mathrm{s}^{-1}\right)$. Quantification of these indices was performed on average over the entirety of the tracked radiations (Dubois et al., 2006) for FA, $\lambda_{\perp}$, and $\lambda_{/ /}$.

VEP recording. The VEP examination was conducted in the same infants a few days after the MRI acquisition, as part of a face perception study (Gliga and Dehaene-Lambertz, 2007).

Data acquisition. Infants passively viewed black and white face images projected on a large screen for $1500 \mathrm{~ms}$, whereas EEG was recorded using a high-density system (65 electrodes; Electrical Geodesics, Eugene, OR) referenced to the vertex and with a $250 \mathrm{~Hz}$ sampling frequency.

Data postprocessing and analysis. The EEG signal was segmented into epochs ( -200 to $1200 \mathrm{~ms}$ ), and both channels and epochs contaminated by motion artifacts were automatically rejected (Gliga and DehaeneLambertz, 2007). The remaining trials (mean, 75 per subject) were digitally filtered between 0.5 and $20 \mathrm{~Hz}$, baseline corrected, averaged, and transformed into reference-independent values using the average reference method. The resulting potential was averaged across a group of posterior electrodes surrounding $\mathrm{Oz}$, and the latency between the image onset and the maximum of the first positive wave (P1) was measured.

Relationships between DTI and VEP measurements. We designed a linear model to quantitatively link DTI indices in the optic radiations and P1 latency during this developmental period. To account for the fact that P1 latency increases with head growth and the distance that signals must travel in the visual pathways, we calculated an "apparent conduction speed" as the ratio between the length of optic pathways (estimated by the distance between the eyes and the occipital poles on T2 images) and P1 latency. In this small age range, the conduction speed was assumed to depend linearly on age and myelination: speed $=$ distance $/ \mathrm{p}_{1}=\alpha$ age + $\beta$-myelination $+\gamma$, where $\alpha, \beta$, and $\gamma$ are adjustable coefficients. The "myelination" term was successively substituted by each DTI index measured in the $3 \mathrm{D}$ reconstructed optic radiations (FA, $\lambda_{\perp}$, and $\lambda_{/ /}$) to test which of these indices significantly affected speed, regardless the effect of age.

To verify that significant correlations between conduction speed and DTI indices were specifically related to maturation of the optic radiations rather than to global maturation processes, we also tested this model in all white matter bundles that can be reliably reconstructed by tractography in infants (corpus callosum, spinothalamic and corticospinal tracts, anterior limb of the internal capsule, external capsule, arcuate, inferior longitudinal and uncinate fascicles, cingulum, and fornix) (Dubois et al., 2008). We computed this analysis for FA and $\lambda_{\perp}$ only, because $\lambda_{/ /}$in the optic radiations did not show any correlation with speed (see Results) and is not generally thought to be related to myelination.

Spatial analysis of DTI indices changes within the optic radiations. We further investigated changes in FA and $\lambda_{\perp}$ along the optic radiations across the infant group. To take into account geometry-based effects, we calculated normalized indices (indicated as $\mathrm{nFA}$ and $\mathrm{n} \lambda_{\perp}$ ) by comparison with an adult group (in which FA is high and $\lambda_{\perp}$ is low): nFA and $\mathrm{n} \lambda_{\perp}$ become close to 1 with maturation through an increase in nFA and a decrease in $\mathrm{n} \lambda_{\perp}$ (Dubois et al., 2008). These normalized indices were then studied by three different methods. First, their averages were computed over three arbitrary segments of the optic radiations (anterior, middle, and posterior) (see Fig. 1a), which were compared two by two over the infant group using two-tailed paired $t$ tests. Second, we evaluated age-related changes in the different segments using linear regression. Finally, to avoid the bias of arbitrary segmentation of the tract, the spatial 
a. Model with age and fractional anisotropy. $R^{2}=0.85$
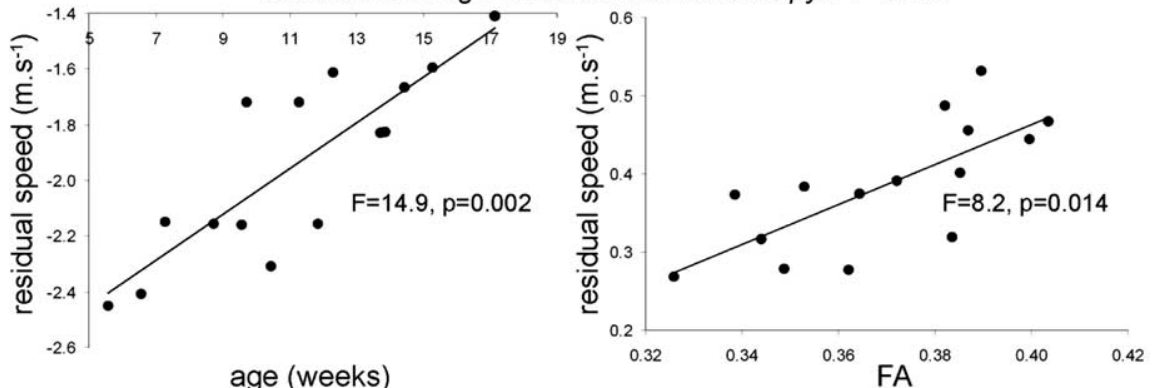

b. Model with age and transverse diffusivity. $R^{2}=0.86$.
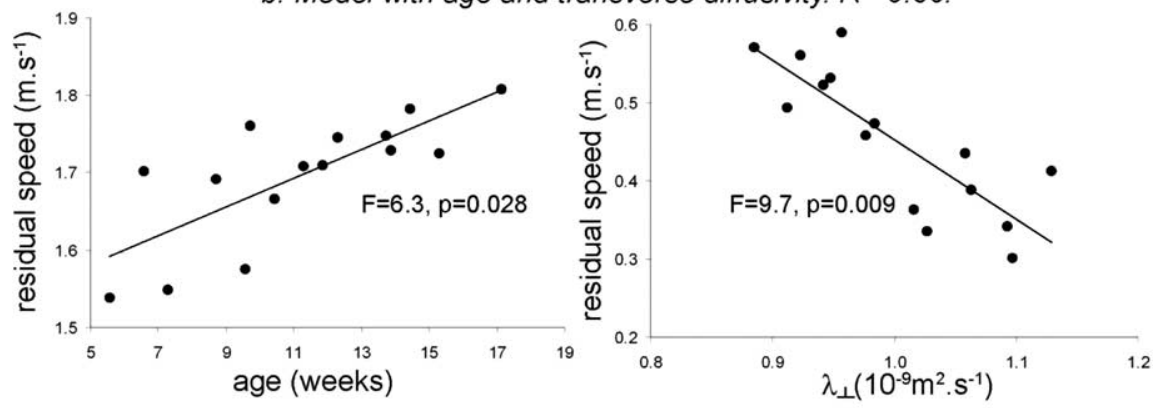

c. Model with age and longitudinal diffusivity. $R^{2}=0.77$.
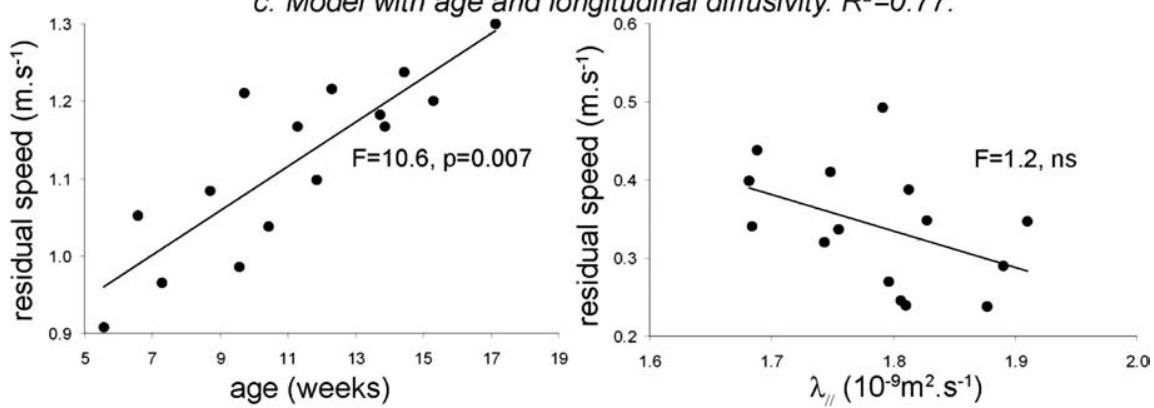

Figure 2. Changes in conduction speed across the infant group with age and DTI indices in the optic radiations. $\boldsymbol{a}-\boldsymbol{c}$, For the models detailed in Table 1 , with age and DTI index covariates (FA, $\left.\lambda_{\perp}, \lambda_{/}\right)$, the plots represent the variations of residual speed, as a function of age after correction for the DTI index effect (left column, speed - $\beta$ DTI index) and as a function of DTI index after correction for the age effect (right column, speed - $\alpha$ age). The contribution of the DTI index was significant for FA and $\lambda_{\perp}$, but not for $\lambda_{/ /}$ns, Not significant.

variations of maturation along the tract were confirmed by precisely evaluating the indices from the LGN to the occipital pole using an interpolated curvilinear abscissa (Gong et al., 2005).

\section{Results}

FA and $\lambda_{\perp}$ reflect VEP functional maturation and fiber myelination

Despite incomplete myelination, the optic radiations and all white matter bundles were reliably reconstructed in infants with DTI-based tractography according to anatomical landmarks (Fig. 1a) (Dubois et al., 2008). P1 latency was identified from the VEP signal (Fig. 1b) (median, $140 \mathrm{~ms}$; range, 120-240 ms).

In the optic radiations, the percentage of variance $\left(R^{2}\right)$ of the conduction speed explained by the models with age and DTI indices as covariates were highly significant (Fig. 2, Table 1). As expected, age contributed significantly to all models, but DTI indices showed variable contributions, with major contributions of FA and $\lambda_{\perp}$, but not $\lambda_{/ /}$. This showed that, in addition to the effect of age, the functional improvement in the efficiency of electrical conduction strongly correlated with changes in FA and $\lambda_{\perp}$.

This correlation between speed and DTI indices was specific to the optic radiations and not an effect of global brain maturation, as demonstrated by the models for all other white matter bundles (Table 1). The contribution of FA and $\lambda_{\perp}$ was nonsignificant compared with age, with the exception of FA in the anterior limb of the internal capsule and in the external capsule, which indicated that $\lambda_{\perp}$ was a more reliable marker of myelination than FA.

\section{Spatial progression of myelination within the optic radiations assessed by DTI}

The comparison of the normalized FA and $\lambda_{\perp}$ indices computed in the anterior, middle, and posterior segments of the optic radiations revealed that myelination was asynchronous over the bundle. First, the anterior segment matured earliest (Fig. $3 a$ ), with the highest nFA (anterior vs middle, $t=6.2, p<0.001$; anterior vs posterior, $t=3.4, p=0.005)$ and lowest $\mathrm{n} \lambda_{\perp}$ (anterior vs middle, $t=-11.8, p<0.001$; anterior vs posterior, $t=-3.0, p=0.009$ ), whereas the middle segment was the least mature, with the highest $\mathrm{n} \lambda_{\perp}$ (middle vs posterior, $t=5.2, p<0.001)$. Second, their changes with age varied across segments (Fig. 3b): significant linear decreases in $n \lambda_{\perp}$ with age were observed in all three segments (anterior, $R^{2}=0.58$, $F=18.1, p=0.001 ;$ middle, $R^{2}=0.56$, $F=16.7, p=0.001$; posterior, $R^{2}=0.45$, $F=10.5, p=0.007$ ), whereas an increase in nFA was only detected in the posterior segment (posterior, $R^{2}=0.48, F=12.2$, $p=0.004)$. Thus, during the first weeks of life, myelination occurs across the whole of the optic radiations but is more advanced in the anterior segment, closest to the LGN, with the highest $\mathrm{nFA}$ and lowest $\mathrm{n} \lambda_{/ /}$, and more intense in the posterior segment, closest to the cortex, with greatest increase of nFA with age.

Finally, these observations were confirmed by the quantification of $\mathrm{nFA}$ and $\mathrm{n} \lambda_{\perp}$ along the optic radiations (Fig. $3 c$ ). To interpret the graphs, note that $\mathrm{nFA}$ values are $<1$ and $\mathrm{n} \lambda$, values are $>1$ in immature tracts, with both indices approaching 1 as the tracts approach the mature state. Beyond the global progression of maturation with age, the mean curve over infants showed the anterior portion matured earlier relative to other regions. With age, the shape of the curve changed mostly in the posterior portion and revealed a "humped" pattern because of the lower nFA and higher $\mathrm{n} \lambda_{\perp}$ of the less mature middle portion. This pattern was particularly evident in the oldest infants (Fig. $3 c$, compare the curves of three infants of different ages) and suggested that the posterior region had the fastest progression of maturation during this developmental period, catching up with the anterior region by 4 months of age.

\section{Discussion}

In this study, we evaluated the microstructural and functional maturation of the visual system using DTI and ERPs during the first postnatal weeks of healthy infancy. In addition to the effect of age, the interindividual differences in P1-based conduction speed 
Table 1. Conduction speed models as function of age and DTI indices in the different bundles

\begin{tabular}{|c|c|c|c|c|c|c|}
\hline \multirow[b]{2}{*}{ Bundles spatial location } & \multicolumn{3}{|c|}{ Fractional anisotropy } & \multicolumn{3}{|c|}{ Transverse diffusivity } \\
\hline & Model fit & Covariate: age & Covariate: FA & Model fit & Covariate: age & Covariate: $\lambda \perp$ \\
\hline Optic radiations ${ }^{a}$ & $R^{2}=0.85$ & $\begin{array}{l}F=14.9 \\
p=0.002\end{array}$ & $\begin{array}{l}F=8.2 \\
p=0.014\end{array}$ & $R^{2}=0.86$ & $\begin{array}{l}F=6.3 \\
p=0.028\end{array}$ & $\begin{array}{l}F=9.7 \\
p=0.009\end{array}$ \\
\hline Corpus callosum & $R^{2}=0.75$ & $\begin{array}{l}F=23.8 \\
p<0.001\end{array}$ & $\begin{array}{l}F=0.3 \\
\text { NS }\end{array}$ & $R^{2}=0.75$ & $\begin{array}{l}F=12.8 \\
p=0.004\end{array}$ & $\begin{array}{l}F=0.1 \\
\text { NS }\end{array}$ \\
\hline Spinothalamic tract & $R^{2}=0.75$ & $\begin{array}{l}F=16.9 \\
p=0.001\end{array}$ & $\begin{array}{l}F=0.1 \\
\text { NS }\end{array}$ & $R^{2}=0.75$ & $\begin{array}{l}F=18.8 \\
p=0.001\end{array}$ & $\begin{array}{l}F=0.0 \\
\text { NS }\end{array}$ \\
\hline Corticospinal tract & $R^{2}=0.75$ & $\begin{array}{l}F=11.4 \\
p=0.005\end{array}$ & $\begin{array}{l}F=0.2 \\
\text { NS }\end{array}$ & $R^{2}=0.76$ & $\begin{array}{l}F=12.5 \\
p=0.004\end{array}$ & $\begin{array}{l}F=1.0 \\
\text { NS }\end{array}$ \\
\hline Anterior limb of the internal capsule & $R^{2}=0.84$ & $\begin{array}{l}F=25.1 \\
p<0.001\end{array}$ & $\begin{array}{l}F=7.1 \\
p=0.020\end{array}$ & $R^{2}=0.77$ & $\begin{array}{l}F=17.7 \\
p=0.001\end{array}$ & $\begin{array}{l}F=1.1 \\
\text { NS }\end{array}$ \\
\hline External capsule & $R^{2}=0.82$ & $\begin{array}{l}F=46.5 \\
p<0.001\end{array}$ & $\begin{array}{l}F=5.4 \\
p=0.038\end{array}$ & $R^{2}=0.75$ & $\begin{array}{l}F=20.5 \\
p=0.001\end{array}$ & $\begin{array}{l}F=0.4 \\
\text { NS }\end{array}$ \\
\hline Arcuate fasciculus & $R^{2}=0.75$ & $\begin{array}{l}F=35.6 \\
p<0.001\end{array}$ & $\begin{array}{l}F=0.2 \\
\text { NS }\end{array}$ & $R^{2}=0.75$ & $\begin{array}{l}F=33.0 \\
p<0.001\end{array}$ & $\begin{array}{l}F=0.4 \\
\text { NS }\end{array}$ \\
\hline Inferior longitudinal fasciculus & $R^{2}=0.77$ & $\begin{array}{l}F=38.9 \\
p<0.001\end{array}$ & $\begin{array}{l}F=1.0 \\
\text { NS }\end{array}$ & $R^{2}=0.76$ & $\begin{array}{l}F=37.3 \\
p<0.001\end{array}$ & $\begin{array}{l}F=0.8 \\
\text { NS }\end{array}$ \\
\hline Uncinate fasciculus & $R^{2}=0.78$ & $\begin{array}{l}F=25.6 \\
p<0.001\end{array}$ & $\begin{array}{l}F=2.2 \\
\text { NS }\end{array}$ & $R^{2}=0.76$ & $\begin{array}{l}F=15.0 \\
p=0.002\end{array}$ & $\begin{array}{l}F=0.7 \\
\text { NS }\end{array}$ \\
\hline Cingulum & $R^{2}=0.75$ & $\begin{array}{l}F=26.5 \\
p<0.001\end{array}$ & $\begin{array}{l}F=0.2 \\
\text { NS }\end{array}$ & $R^{2}=0.76$ & $\begin{array}{l}F=23.2 \\
p<0.001\end{array}$ & $\begin{array}{l}F=0.6 \\
\text { NS }\end{array}$ \\
\hline Fornix & $R^{2}=0.77$ & $\begin{array}{l}F=16.2 \\
p=0.002\end{array}$ & $\begin{array}{l}F=1.5 \\
\text { NS }\end{array}$ & $R^{2}=0.76$ & $\begin{array}{l}F=14.2 \\
p=0.003\end{array}$ & $\begin{array}{l}F=0.9 \\
\text { NS }\end{array}$ \\
\hline
\end{tabular}

The bundle spatial location for DTI quantification, the percentage of variance explained by the model $\left(R^{2}\right)$, and the statistical significance ( $F$ and $p$ values; NS, not significant at the level of $\left.p<0.05\right)$ for both covariates (age and DTI index $\mathrm{FA} / \lambda_{\perp}$ ) are outlined. The highest $R^{2}$ values were measured for the models with FA and $\lambda_{\perp}$ in the optic radiations.

${ }^{a}$ For the model of speed as a function of age and longitudinal diffusivity in the optic radiations: $R^{2}=0.77 ;$ age, $F=10.6 ; p=0.007 ; \lambda /, F=1.2 ; \mathrm{NS}$.

were strongly correlated with DTI indices in the optic radiations only, demonstrating that FA and $\lambda_{\perp}$, but not $\lambda_{/ /}$, are structural markers of myelination, with $\lambda_{\perp}$ being a stronger marker than $\mathrm{FA}$, and that changes in FA and $\lambda_{\perp}$ are closely related to a functional improvement in the efficiency of electrical conduction. Using fiber tractography and an original quantitative analysis of normalized FA and $\lambda_{\perp}$, we revealed an asynchronous progression of two fronts of myelination along the optic radiations.

The latency of P1 wave is the classical parameter used to evaluate visual perception development in human infants, because it is robustly present from birth on, unlike the shorter negative component (Kraemer et al., 1999; Lippe et al., 2007). Although we used face stimuli instead of the more classical checkerboards or flashes, the age-related variations in P1 latency that were measured were consistent with previous results (McCulloch et al., 1999), implying that our functional measure was a good marker of the first stages of visual processing [see the study by Gliga and Dehaene-Lambertz (2005) showing similar P1 latencies for faces and scrambled images]. Although the observed shortening in P1 latency with age could be caused by several factors (such as retinal development, myelination of the retinotectal pathway, neuronal maturation, or synaptogenesis in the LGN and visual cortex), the myelination of optic radiations has a major role in the speeding up of this electrophysiological component.

With DTI-based tractography, the optic radiations could be reliably segmented in the immature infant brain (Dubois et al., 2008) by using a nonlinear algorithm particularly robust to low FA in the place of fiber crossings (Perrin et al., 2005). Although DTI indices can be affected by partial volume effects, crossing fibers or increases in the compactness of the bundles, these incidental factors do not influence electrical transmission. The significant correlations between visual conduction speed and DTI indices in the optic radiations regardless the effect of age demonstrated that FA and, even more strongly, $\lambda_{\perp}$ changes can characterize the functional improvement in electrical conduction and represent structural markers of myelination. Furthermore, the models with DTI indices in the other white matter bundles ruled out an effect of global brain maturation on the increased speed of P1 component, demonstrated its specificity to the geniculooccipital pathway, and indicated that $\lambda_{\perp}$ is a more reliable marker of myelination than FA.

Recently, associations between white matter maturation evaluated by DTI and the development of cognitive functions have been observed during childhood (Nagy et al., 2004), and variations in FA have been associated with behavioral performance on measures such as reading scores or short-term verbal memory capacities (Niogi and McCandliss, 2006), underscoring the importance of brain connectivity for efficient cognitive processes. However, to our knowledge, this is the first time that a direct relationship between quantitative structural indices (FA and $\lambda_{\perp}$ ) and a functional parameter (P1 latency) has been demonstrated in living infants. Our observation of a close correlation between changes in FA and $\lambda_{\perp}$ and conduction speed demonstrates that these indices can provide a faithful approximation of white matter functional maturation and might be used to test functions that are not easily testable in young infants (e.g., motor functions) or that are not fully developed (e.g., long-range connectivity).

The quantification of normalized FA and $\lambda_{\perp}$ revealed the heterogeneous and asynchronous maturation of the optic radiations, with progression in the anterior, then posterior, and finally middle regions. Maturation of the anterior segment was relatively advanced and stable across the entire age range (5.6-17.1 weeks of age), whereas major changes occurred in the posterior segment, which catches up with the anterior segment over this developmental period, revealing a "humped" pattern in the oldest infants. Whereas crossing fibers or partial volume effects with surrounding tissue and corticospinal fluid at the level of the ventricles may affect DTI indices, their heterogeneity along the optic radiations may hardly be attributed to such factors, because we compared indices normalized for adult values after adjustment for image spatial resolution (Dubois et al., 2008), and we checked that the bundles were precisely isolated in the infant brain as in 


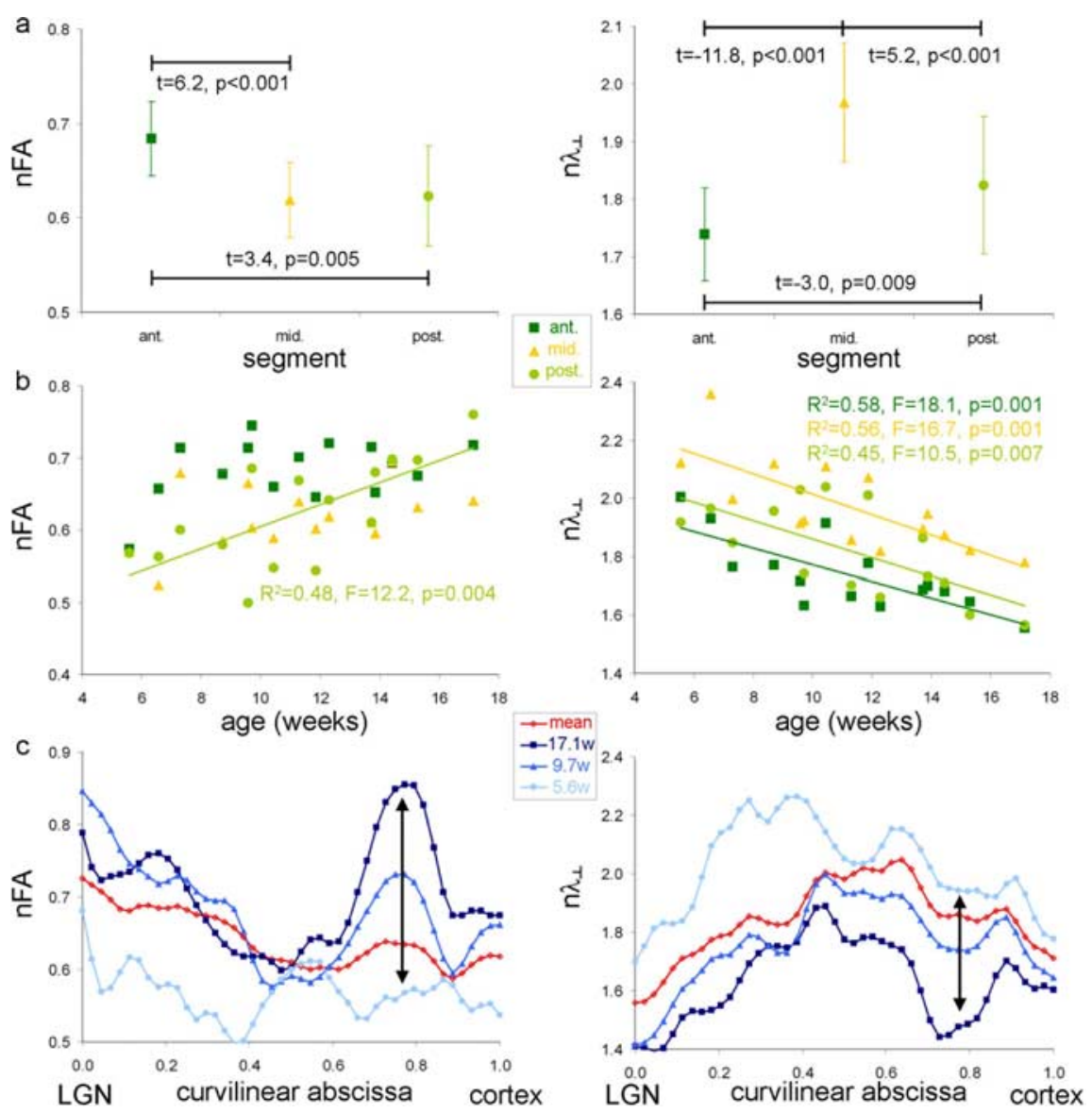

Figure 3. Heterogeneous maturation within and along the optic radiations. Left and right columns represent normalized FA and $\lambda_{\perp}$, respectively. $\boldsymbol{a}$, Group mean indices, with quantification on average over the three segments (with SDs in plot bars). Advanced maturation was observed in the anterior segment, with indices closest to 1, followed by the posterior segment, with lower $\mathrm{n} \lambda_{\perp}$ compared with the middle segment. ant, Anterior; mid, middle; post, posterior. $\boldsymbol{b}$, Age-related changes of indices, showing an increase in $n F A$ in the posterior segment and decreases in $n \lambda_{\perp}$ in all segments. $c$, Quantification of normalized indices along the tracts, from the LGN (abscissa, 0 ) to the occipital pole (abscissa, 1): mean over the infant group and results for subjects of different ages $(5.6,9.7$, and 17.1 weeks of age). The topographical patterns were relatively similar across infants (normalized indices approach 1 with age and maturation), except at the level of the posterior segment where the interindividual variability was high (arrows), with a strong increase of $\mathrm{nFA}$ and decrease of $\mathrm{n} \lambda_{\perp}$ with age.

the adult brain. This DTI pattern rather suggests heterogeneity in maturation, as reported previously for the corticospinal tract (McArdle et al., 1987), with progression along both fronts of the optic radiations. According to the hypothesis that myelination proceeds from the neuron body to the periphery (McCart and Henry, 1994), it may result from the myelination of both geniculocortical-bound (projection) and corticogeniculatebound (feedback) fibers within the optic radiations. In the youngest infants, the delay of the corticogeniculate fibers compared with the geniculocortical fibers would reflect the delayed maturation of the cortical retrocontrol to the thalamus relative to bottom-up fibers. Indeed, the development of an efficient feedback transmission might contribute to the rapid changes observed in face recognition capacities, notably after 2 months of age (de Haan, 2001).

With our approach, it would be interesting to study older infants as P1 latency reaches its asymptotic adult value by 20 weeks of age. Beyond that point, the ongoing myelination should compensate for the continuous brain growth. Recently, such a process has been demonstrated in the adult mouse (Salami et al., 2003): the differential myelination enables the modulation of the electrical conduction speed and keeps the conduction time be- tween the LGN and different regions of the primary visual cortex constant, despite the differing lengths of the various fibers, so that visual information treatment remains simultaneous for all cortical neurons. Studying infants with peripheral visual impairment would further help to investigate the role of external stimulation on structural maturation, because animal studies have demonstrated that neuronal activity induced by stimulation influences the degree of white matter myelination (Gyllensten and Malmfors, 1963; Tauber et al., 1980; Barres and Raff, 1993; Demerens et al., 1996).

\section{Conclusion}

By combining two complementary noninvasive imaging modalities, we were able to demonstrate a direct quantitative relationship between the microstructural maturation of the optic radiations and the functional development of visual perception during early infancy. With DTI and tractography, we were able to dissect the optic radiations into three segments with asynchronous time courses, suggesting two fronts of myelination in both the corticogeniculate and the geniculocortical fibers. DTI thus appears to be a new functional tool to estimate electrical conduction along white matter tracts, to explore early developmental impairments, and to understand mechanisms of plasticity that support functional reorganization.

\section{References}

Barres BA, Raff MC (1993) Proliferation of oligodendrocyte precursor cells depends on electrical activity in axons. Nature 361:258-260.

Beaulieu C (2002) The basis of anisotropic water diffusion in the nervous system-a technical review. NMR Biomed 15:435-455.

Benavente I, Tamargo P, Tajada N, Yuste V, Olivan MJ (2005) Flash visually evoked potentials in the newborn and their maturation during the first six months of life. Doc Ophthalmol 110:255-263.

Catani M, Howard RJ, Pajevic S, Jones DK (2002) Virtual in vivo interactive dissection of white matter fasciculi in the human brain. NeuroImage 17:77-94.

Chiron C, Raynaud C, Mazière B, Zilbovicius M, Laflamme L, Masure MC, Dulac O, Bourguignon M, Syrota A (1992) Changes in regional cerebral blood flow during brain maturation in children and adolescents. J Nucl Med 33:696-703.

Chugani HT, Phelps ME (1986) Maturational changes in cerebral function in infants determined by $18 \mathrm{FDG}$ positron emission tomography. Science 231:840-843.

Cointepas Y, Poupon C, Maroy R, Riviere D, Le Bihan D, Mangin JF (2003) A freely available Anatomist/BrainVISA package for analysis of diffusion MR data. Proceedings of the 9th HBM Scientific Meeting, New York. NeuroImage 19:S810.

Crognale MA, Kelly JP, Chang S, Weiss AH, Teller DY (1997) Development of pattern visual evoked potentials: longitudinal measurements in human infants. Optom Vis Sci 74:808-815.

de Haan MD (2001) The neuropsychology of face processing during infancy and childhood. In: Handbook of developmental cognitive neuroscience (Nelson CA, Luciana M, eds), pp 381-398. Cambridge, MA: Bradford.

Demerens C, Stankoff B, Logak M, Anglade P, Allinquant B, Couraud F, Zalc 
B, Lubetzki C (1996) Induction of myelination in the central nervous system by electrical activity. Proc Natl Acad Sci USA 93:9887-9892.

Dubois J, Hertz-Pannier L, Dehaene-Lambertz G, Cointepas Y, Le Bihan D (2006) Assessment of the early organization and maturation of infants' cerebral white matter fiber bundles: a feasibility study using quantitative diffusion tensor imaging and tractography. NeuroImage 30:1121-1132.

Dubois J, Dehaene-Lambertz G, Perrin M, Mangin JF, Cointepas Y, Duchesnay E, Le Bihan D, Hertz-Pannier L (2008) Asynchrony of the early maturation of white matter bundles in healthy infants: quantitative landmarks revealed non-invasively by diffusion tensor imaging. Hum Brain Mapp 29:14-27.

Gliga T, Dehaene-Lambertz G (2005) Structural encoding of body and face in human infants and adults. J Cogn Neurosci 17:1328-1340.

Gliga T, Dehaene-Lambertz G (2007) Development of a view-invariant representation of the human head. Cognition 102:261-288.

Gong G, Jiang T, Zhu C, Zang Y, Wang F, Xie S, Xiao J, Guo X (2005) Asymmetry analysis of cingulum based on scale-invariant parameterization by diffusion tensor imaging. Hum Brain Mapp 24:92-98.

Gyllensten L, Malmfors T (1963) Myelinization of the optic nerve and its dependence on visual function-a quantitative investigation in mice. J Embryol Exp Morphol 11:255-266.

Huttenlocher PR, Dabholkar AS (1997) Regional differences in synaptogenesis in human cerebral cortex. J Comp Neurol 387:167-178.

Huttenlocher PR, de Courten C, Garey LJ, Van der Loos H (1982) Synaptogenesis in human visual cortex-evidence for synapse elimination during normal development. Neurosci Lett 33:247-252.

Kos-Pietro S, Towle VL, Cakmur R, Spire JP (1997) Maturation of human visual evoked potentials: 27 weeks conceptional age to 2 years. Neuropediatrics 28:318-323.

Kraemer M, Abrahamsson M, Sjostrom A (1999) The neonatal development of the light flash visual evoked potential. Doc Ophthalmol 99:21-39.

Le Bihan D, Mangin JF, Poupon C, Clark CA, Pappata S, Molko N, Chabriat H (2001) Diffusion tensor imaging: concepts and applications. J Magn Reson Imag 13:534-546.

Lippe S, Roy MS, Perchet C, Lassonde M (2007) Electrophysiological markers of visuocortical development. Cereb Cortex 17:100-107.

McArdle CB, Richardson CJ, Nicholas DA, Mirfakhraee M, Hayden CK, Amparo EG (1987) Developmental features of the neonatal brain: MR imaging. Part I. Gray-white matter differentiation and myelination. Radiology 162:223-229.

McCart RJ, Henry GH (1994) Visual corticogeniculate projections in the cat. Brain Res 653:351-356.
McCulloch DL, Orbach H, Skarf B (1999) Maturation of the patternreversal VEP in human infants: a theoretical framework. Vision Res 39:3673-3680.

Mori S, van Zijl PC (2002) Fiber tracking: principles and strategies-a technical review. NMR Biomed 15:468-480.

Nagy Z, Westerberg H, Klingberg T (2004) Maturation of white matter is associated with the development of cognitive functions during childhood. J Cogn Neurosci 16:1227-1233.

Neil JJ, Miller J, Mukherjee P, Hüppi PS (2002) Diffusion tensor imaging of normal and injured developing human brain-a technical review. NMR Biomed 15:543-552.

Niogi SN, McCandliss BD (2006) Left lateralized white matter microstructure accounts for individual differences in reading ability and disability. Neuropsychologia 44:2178-2188.

Partridge SC, Mukherjee P, Henry RG, Miller SP, Berman JI, Jin H, Lu Y, Glenn OA, Ferriero DM, Barkovich AJ, Vigneron DB (2004) Diffusion tensor imaging: serial quantitation of white matter tract maturity in premature newborns. NeuroImage 22:1302-1314.

Paus T, Collins DL, Evans AC, Leonard G, Pike B, Zijdenbos A (2001) Maturation of white matter in the human brain: a review of magnetic resonance studies. Brain Res Bull 54:255-266.

Perrin M, Poupon C, Cointepas Y, Rieul B, Golestani N, Pallier C, Riviere D, Constantinesco A, Le Bihan D, Mangin JF (2005) Fiber tracking in $\mathrm{q}$-ball fields using regularized particle trajectories. Proceedings of the IPMI Scientific Meeting, Glenwood Springs, CO, July.

Salami M, Itami C, Tsumoto T, Kimura F (2003) Change of conduction velocity by regional myelination yields constant latency irrespective of distance between thalamus and cortex. Proc Natl Acad Sci USA 100:6174-6179.

Song SK, Sun SW, Ju WK, Lin SJ, Cross AH, Neufeld AH (2003) Diffusion tensor imaging detects and differentiates axon and myelin degeneration in mouse optic nerve after retinal ischemia. NeuroImage 20:1714-1722.

Tauber H, Waehneldt TV, Neuhoff V (1980) Myelination in rabbit optic nerves is accelerated by artificial eye opening. Neurosci Lett 16:235-238.

Tsuneishi S, Casaer P (1997) Stepwise decrease in VEP latencies and the process of myelination in the human visual pathway. Brain Dev 19:547-551.

Yakovlev PI, Lecours AR (1967) The myelogenetic cycles of regional maturation in the brain. In: Regional development of the brain in early life (Minowski A, ed), pp 3-68. Oxford: Blackwell. 